\title{
Using the lens of phonetic experience to resolve phonological forms
}

\author{
Catherine T. Best ${ }^{\mathrm{a}, \mathrm{b}}$, Ann R. Bradlow ${ }^{\mathrm{c}}$, Susan Guion-Anderson ${ }^{\mathrm{d}, *}$, Linda Polka ${ }^{\mathrm{e}}$ \\ a MARCS Auditory Laboratories, University of Western Sydney, Australia \\ ${ }^{\mathrm{b}}$ Haskins Laboratories, New Haven, CT, USA \\ c Linguistics Department, Northwestern University, Evanston, IL, USA \\ d Linguistics Department, University of Oregon, Eugene, OR, USA \\ e School of Communication Sciences E Disorders, McGill University, Montreal, Canada
}

\begin{abstract}
A B S T R A C T
This special issue of the Journal contains a selection of papers developed from original presentations at the 2nd ASA Special Workshop on Speech with the theme of Cross-Language Speech Perception and Variations in Linguistics Experience. The papers represent major theoretical and empirical contributions that converge upon the common theme of how our perception of phonological forms is guided and constrained by our experience with the phonetic details of the language(s) we have learned. Several of the papers presented here offer key theoretical advances and lay out novel or newly expanded frameworks that increase our understanding of speech perception as shaped by universal, first language acquisition abilities, general learning mechanisms, and language-specific perceptual tuning. Others offer careful empirical investigations of language learning by simultaneous bilinguals, as well as by later second language learners, and discuss their new findings in light of the theoretical proposals. The work presented here will provide a stimulating and thoughtful impetus toward further progress on the fundamentally significant issue of understanding of how language experience shapes our perception of phonetic details and phonological structure in spoken language.
\end{abstract}

(c) 2011 Elsevier Ltd. All rights reserved.

In May 2009, the Acoustical Society of America sponsored the 2nd Special Workshop on Speech with the theme of CrossLanguage Speech Perception and Variations in Linguistics Experience, in Portland, Oregon, USA. ${ }^{1}$ The Workshop addressed new theoretical and empirical directions in research on the effects of experience on speech perception. Topics discussed ranged from behavioral and neural effects in adults to their development in young children. The presentations covered a wide array of types of language experience, including listeners naïve to the stimulus languages, as well as those learning the target language as a second language (L2 learners), and those already quite proficient in two or more languages from an early age (simultaneous and early sequential bilinguals). The venue was designed to foster discussion and further progress in understanding how phonetic experience shapes listeners' ability to apprehend phonological structure, by bringing together established and early career researchers whose work represents recent advances and new synergies in theory and research at the dynamic intersection of

\footnotetext{
* Corresponding author. Tel.: +1 541346 3682; fax: +1 543465961.

E-mail address: guion@uoregon.edu (S. Guion-Anderson).

${ }^{1}$ The sponsors also included MARCS Auditory Laboratories (University of Western Sydney), the University of Oregon, the Centre for Research on Language, Mind and Brain (McGill University), Northwestern University, and Haskins Laboratories.
}

speech perception and linguistic experience. The scientific program of the Workshop covered 2.5 days, comprising 147 presentations, including ten invited talks, ten contributed talks, a special address at the banquet, a closing address, and a total of 124 posters organized into three sessions. A special keynote address was given by Winifred Strange, who also received the Acoustical Society of America's Silver Medal in Speech Communication at the May 2009 main meeting of the Acoustical Society.

The papers in this special issue of the Journal of Phonetics represent several major theoretical and empirical contributions that arose from the foundations laid by the Workshop. As indicated by the title of this introductory paper, these contributions converge from a variety of directions upon the common theme of how our perception of phonological forms - phonemes, contrasts, words - is guided and constrained by our experience with the phonetic details of the language(s) we have learned. Importantly, the papers presented in this special edition go well beyond the content of the Workshop's invited and contributed presentations and ensuing discussions. As such, the collection attests to the meeting's success in fostering further interactions among the contributors and facilitating additional blossoming of the topics covered at the Workshop. Several of the papers presented here offer key theoretical advances and lay out novel or newly expanded frameworks that increase our understanding of speech perception as shaped by universal, first language 
acquisition abilities, general learning mechanisms, and languagespecific perceptual tuning. Others offer careful empirical investigations of language learning by simultaneous bilinguals, as well as by later second language learners, and discuss their new findings in light of the theoretical proposals.

The first set of papers present new theoretical and empirical advances on the topics of speech perception and word recognition in native and non-native listeners. This section begins with the centerpiece of this special issue, an expanded version of the Workshop's keynote address by Winifred Strange ("Automatic Selective Perception (ASP) of first and second language speech: A working model"). This paper presents a model of speech perception that aims to explain why native and non-native language listening are fundamentally different activities for listeners. A key insight incorporated into this model is the distinction between automatic versus attentional processing, which represent opposite ends of a continuum of processing effort for both native and non-native listeners. Task-, stimulus- and listener-specific factors determine whether a particular instance of speech perception will involve primarily automatic or attentional processing, or some balance between the two extremes. In this model, the key difference between native and non-native listening is in terms of access to selective perception routines that underlie automatic processing. While native listeners can enjoy the benefit of selective perception routines that have been optimized to the given language and operate on the automatic end of the continuum of effort, non-native listeners are hindered by selective perception routines developed for their first language (L1) that may be non-optimal with respect to the target language. Therefore, they are forced to operate at the attentional end of the continuum of effort. In her contribution, Strange discusses the details of this model and presents a wealth of supporting data from her work with her many associates over almost four decades. It is our hope and belief that this simultaneously retrospective and forward-looking contribution will be seminal in the field of crosslanguage and second-language speech perception, a field in which Winifred Strange has been a "game changer" from the very beginning.

The paper by Linda Polka and Ocke-Schwen Bohn ("Natural Referent Vowel (NRV) framework: An emerging view of early phonetic development") formally introduces a framework for understanding early phonetic development, specifically with respect to vowel perception. The authors had previously reported an underlying bias in infants' vowel discrimination, which had led them to posit that peripheral vowels may serve as universal perceptual reference points that aid infants in learning the layout of their native language vowel space (Polka \& Bohn, 2003). Here, they provide a thorough update and discussion of more recent empirical findings on infant and adult vowel discrimination, for both native and non-native vowel contrasts and for a variety of L1s, taken from their own and other laboratories. The full body of evidence overwhelmingly supports their premise that vowels falling near the periphery of a universal vowel space act as perceptual referent vowels that shape learning of the vowel systems, whether or not the target vowels occur in the listener's own language. The insightful discussion of how the NRV framework may relate to linguistic typologies and to other existing models of speech perception highlights its promise as a conceptual tool for improving our understanding of the ways in which universal biases and experience-dependent learning combine to shape phonetic development.

The contribution by Andrea Weber, Mirjam Broersma and Makiko Aoyagi ("Spoken word recognition in foreign-accented speech by L2 listeners") asks how spoken word recognition proceeds when non-native (L2) listeners encounter foreignaccented speech. This work was motivated by the general goal of understanding how listeners handle signal variability when required to recognize spoken words, and by the particular concern for the increasingly common real-world situations that involve non-native talkers and non-native listeners. This work is representative of increasing interest in cross-language and L2 speech production and perception across the hierarchy of linguistic units from segments to words and sentences. In particular, the study in this paper offers insight into the online processing of words produced by non-native talkers as perceived by non-native listeners. The interesting conclusion of this paper is that, despite their deviation from native talker norms, foreign-accented words can facilitate word recognition for non-native listeners if the listener's native language matches that of the talker or if the acoustic form of the to-be-recognized word is indistinguishable from the listener's standard pronunciation. Thus, this paper demonstrates how the deviations of foreign-accented speech need not present word recognition difficulties for all listeners, an important point to bring to light as we broaden the reach of cross-language and L2 phonetics.

The second set of papers addresses the blossoming area of theory and research on perceptual and neural underpinnings of early bilingual development. In the first paper of this set, Suzanne Curtin, Krista Byers-Heinlein and Janet Werker ("Bilingual beginnings as a lens for theory development: PRIMIR in focus") extend the theoretical model PRIMIR (Processing Rich Information from Multidimensional Interactive Representations), their framework for understanding the relation between experiential tuning of infants' perception of native and non-native speech and emerging recognition of spoken words (Werker \& Curtin, 2005; Curtin \& Werker, 2007), to infants growing up bilingual. The bilingual extension both addresses an important and increasing sector of the population, and simultaneously raises important new considerations for understanding the role of experience in early lexical development. As the authors aptly argue, bilingual language acquisition calls for reconsideration of "task demands," as PRIMIR had been previously applied only to monolingual infants. In light of bilingual infants' more complex language-learning situation, task demands must now be seen as including both external forces imposed by the language environment, and internal factors brought by the infant to the experimental situation. Curtin and colleagues have also added a critical new component to their proposed mechanisms for speech learning, a comparison/contrast mechanism that works together with the previously posited statistical learning mechanism. While the current paper focuses on bilingual infants, the revised PRIMIR framework will certainly expand and deepen our understanding of the relationship between speech perception and word learning in all infants.

Megha Sundara and Adrienne Scutellaro contribute to our understanding of bilingual phonological development in their article ("Rhythmic distance between languages affects the development of speech perception in bilingual infants"). While the field has generally accepted that monolingual children learn phonological categories based on perceptual learning mechanisms which exploit infants' sensitivity to statistical properties of speech input, previous work has suggested that bilingual infants are not able to separate their two languages early on, leading to the inability to perceptually distinguish some phonological contrasts in one or the other language. Sundara and Scutellaro examine the ability of young bilinguals to sort their two languages and track their statistical properties separately using rhythmic properties. They note that previous work with bilingual infants has focused on two languages with similar rhythm and so, in an innovative move, they step beyond that constraint to examine the perception of bilingual infants with rhythmically different languages: English and Spanish. They find that both monolingual and bilingual infants are able to distinguish the English $|e /-| \varepsilon /$ contrast by 8 months of age. These results indicate that bilingual infants are 
able to keep pace with their monolingual peers when they have the same sorts of tracking tools available to them, which may be enhanced when rhythmicity differences allow them to recognize the individuality of their two languages from early on. This finding suggests that we must consider the linguistic circumstances carefully, and in a variety of contexts, before accepting any inherent limitations on bilingual vis-à-vis monolingual development.

Laura Bosch and Marta Ramon-Casas ("Variability in vowel production by bilingual speakers: Can input properties hinder the early stabilization of contrastive categories") focus on the phonetic input experienced by the bilingual infant and in doing so expose an important aspect of the complexity associated with speech development in bilingual acquisition. The authors were motivated to explore input issues to explain their earlier perception findings that bilingual (Catalan-Spanish) infants take longer to build native phonetic categories for some Catalan vowels in comparison to their Catalan monolingual peers (Bosch \& Sebastián-Gallés, 2003). To explore this further Bosch \& RamonCasas take a detailed look at Catalan mid-front vowels produced by two groups of adult bilingual speakers of Spanish and Catalan. All of their speakers were fluent in Catalan and used it in their daily life but differed in their early language acquisition experiences. One group had a consistent exposure to both languages from infancy (BSC group); the other group was exposed to Catalan during infancy and began learning Spanish in the pre-school years (C group). There were some minor differences in the acousticphonetic properties of the $/ \mathrm{e} /$ and $/ \varepsilon /$ Catalan vowels produced by each group. However, a more striking finding was that the BSC group made frequent production errors at the lexical level, producing $/ e /$ in $/ \varepsilon /$ target words. Given the many cognate words in Spanish and Catalan, these errors create a kind of messy input that can be expected to impede an infant learner, which likely explains (in part) the challenge that bilingual infants face in perceptual acquisition of this vowel contrast. Overall, Bosch and Ramon-Casas findings highlight the important role of input in shaping and stabilizing the initial representation of phonetic categories. They also force us to recognize that, at the phonetic level, the input experienced by a monolingual and a bilingual infant acquiring the same language may be qualitatively different in ways that can systematically affect their phonological acquisition.

Valerie Shafer, Yan Yu, and Hia Datta ("The development of English vowel perception in monolingual and bilingual infants: Neurophysiological correlates") explore the development of event-related-potential (ERP) electrophysiological components in monolingual and bilingual infants and children. Their results indicate that overall, bilingual and monolingual infants process the same speech stimuli in different ways. A major finding of the study is that there are differences in responses to English vowel contrasts by monolingual English versus bilingual Spanish-English infants. The monolingual infants showed robust positive discriminative responses (pMMR), while the bilingual infants, especially the females, had a different scalp distribution for these positive responses, as well as more negative responses (nMMR). The authors offer an appealing attentional interpretation of these findings. Specifically, Shafer and colleagues argue that the more negative responses indicate greater attentional allocation to speech processing. On this interpretation, the monolingual infants were using fewer attentional resources to process the stimuli than the bilingual infants. We can relate this finding to the ASP model described by Strange in this volume, by suggesting that because fewer automatic selective perception routines (ASPs) are needed for a monolingual phonological system, the monolingual system may be up and running before the greater number of ASPs that are needed for a bilingual phonological system have all become fully automated. Thus, differences in the time course of development between monolingual and bilingual children can be explained by appealing to the same underlying set of basic learning mechanisms, along with the need for a larger number of such perceptual routines to be established when the child is learning more than one language.

The paper by Adrian Garcia-Sierra, Maritza Rivera-Gaxiola, Cherie Percaccio, Barbara Conboy, Harriett Romo, Lindsay Klarman, Sophia Ortiz and Patricia Kuhl ("Bilingual language learning: An ERP study relating early brain responses to speech, language input, and later word production") breaks new ground by providing the most comprehensive longitudinal study of early bilingual language learning to date. The authors tested infants from bilingual Spanish/English families in two infant age groups (6-9 and 10-12 months) to examine the relationship between estimates of language exposure based on interview data, and neural measures of phonetic perception to a Spanish and an English stop voicing contrast. The predictive relationship between each measure and later word production skills in both languages at 15 months was also assessed. The neural findings suggest that perceptual differentiation of these native contrasts emerges later in bilingual infants, consistent with some previous behavioral findings. At 10-12 months, the neural responses to each contrast reflected the relative exposure level to each language. Language dominance in the neural responses and in the exposure indices assessed in infancy also predicted language dominance in later word production skills. These concurrent and predictive relationships confirm the link between early speech perception and later language skills demonstrated in earlier research, but also show that variation in language exposure profiles impacts the acquisition profiles of bilingual infants. There appears to be a global impact of bilingual exposure that is consistent with the authors' proposal that bilingual infants perceptually adapt to an increased level of phonetic variability by extending the developmental period devoted to perceptual narrowing to the ambient language structure. With this study we arrive one step closer to linking input measures to individual performance, an exciting achievement that will bring new insights to bilingual acquisition and will have a broad impact on theories of language learning.

Taken together, the papers in this special issue provide a key selection of recent advances and current work in the area of speech perception and linguistic experience. Importantly, this collection necessarily reflects just a sampling of the current burgeoning of research on non-native, L2, and bilingual speech perception. Much further theorizing and empirical work is needed over the coming years to refine and elaborate our understanding of speech learning. Progress toward that goal appears to be well underway, with much evidence of increasing interest in crosslanguage and bilingual speech perception and spoken word recognition in our ever-more-global, multilinguistic and multicultural world. This special issue and the workshop on which it was based provide a clear and important step towards achieving a broader and deeper understanding of how language experience shapes our perception of phonetic details and phonological structure in spoken language. We hope the readers of this special issue find the work presented here a stimulating and thoughtful impetus toward further progress on these fundamentally significant issues.

\section{References}

Bosch, L., \& Sebastián-Gallés, N. (2003). Simultaneous bilingualism and the perception of a language-specific vowel contrast in the first year of life. Language and Speech, 46, 217-243.

Curtin, S., \& Werker, J. F. (2007). The perceptual foundation of phonological development. In G. Gaskell (Ed.), The Oxford handbook of psycholinguistics (pp. 579-599). Oxford University Press.

Polka, L., \& Bohn, O.-S. (2003). Asymmetries in vowel perception. Speech Communication, 41, 221-231.

Werker, J. F., \& Curtin, S. (2005). PRIMIR: A developmental model of speech processing. Language Learning and Development, 1(2), 197-234. 